\title{
Integración de las TIC en los procesos de enseñanza- aprendizaje de la especialidad de Pedagogía en los conservatorios superiores de música
}

\section{Integration of ICT in the teaching and learning processes of the specialty of Pedagogy in the superior music conservatories}

\author{
Nuria Díez Latorre \\ Universidad de Lleida \\ ndiezlatorre@gmail.com \\ Xavier Carrera Farran \\ Universidad de Lleida \\ carrera@pip.udl.cat
}

Recibido: $21 / 09 / 2018$

Aceptado: $27 / 11 / 2018$

Publicado: 21/12/2018

\section{RESUMEN}

Esta investigación analiza la integración de Tecnologías de la Información y la Comunicación (TIC) en los procesos de enseñanza-aprendizaje de los futuros pedagogos de música. Para ello se ha tomado en consideración la perspectiva del profesorado de los departamentos de Pedagogía de 14 conservatorios superiores españoles. Los datos se han recogido a partir de un cuestionario y un grupo de discusión. En general la predisposición a utilizar las TIC es alta, pero se basa en criterios de acceso a recursos y eficacia en las tareas docentes más que al fomento de prácticas pedagógicas innovadoras.

\section{PALABRAS CLAVE}

TIC; formación docente; educación musical; conservatorio.

\section{ABSTRACT}

This research analyzes how Information and Communication Technologies (ICT) are integrated into teaching and the learning process of future music pedagogues. For this purpose, integration of ICT and teaching perspective of 14 Spanish superior music conservatories was analyzed through the conduction of a survey and the evaluation of a discussion group. The results suggest that, in general, there is a high predisposition towards the use of ICTs. They also show that this predisposition is based on access to resources and the efficiency in teaching tasks criteria rather than the promotion of innovative pedagogical practices.

\section{KEYWORDS}

ICT; teacher training; musical education; music school. 


\section{CITA RECOMENDADA}

Díez, N y Carrera, X. (2018). Integración de las TIC en los procesos de enseñanza-aprendizaje de la especialidad de Pedagogía en los conservatorios superiores de música. RIITE. Revista Interuniversitaria de Investigación en Tecnología Educativa, 5, 40-55. Doi: http://dx.doi.org/10.6018/riite/2018/342681

\section{Principales aportaciones del artículo y futuras líneas de investigación:}

- Conocer cómo integran las TIC en su docencia los profesores de Pedagogía de los conservatorios superiores de música.

- Ofrecer un punto de partida para futuros diseños de planes de intervención y de formación del profesorado.

- Contribuir al debate sobre la modernización de la enseñanza en los conservatorios de música.

\section{INTRODUCCIÓN}

Las instituciones educativas deben considerar el nuevo escenario de desarrollo tecnológico y repensar lo que supone enseñar música en un mundo digital. En el ámbito de la enseñanza en los conservatorios y escuelas de música se deberían considerar las ventajas y potencialidades que ofrecen las Tecnologías de la Información y la Comunicación (TIC). Sin embargo, como señalan Area (2007) y Serrano (2017), estas no generan mejoras en la enseñanza y el aprendizaje por sí mismas ni conllevan necesariamente procesos de innovación. Las ventajas de las TIC no dependen tanto de sus propias características como del uso que se haga de ellas, de la organización del espacio, del tiempo, de la estrategia metodológica que se siga y de la interacción que se establezca entre el docente y los alumnos. Es decir, que se debe planificar adecuadamente su uso para que realmente resulten beneficiosas y promuevan aprendizajes significativos, lo que depende inevitablemente de la mediación del profesorado.

Es en los conservatorios superiores de música donde adquieren su preparación los pedagogos cuya futura labor docente se desarrollará en escuelas de música y conservatorios elementales y profesionales. Entre otras, se encargarán de impartir durante seis cursos la asignatura de Lenguaje Musical, base de todas las destrezas además de la práctica del instrumento. Los pedagogos musicales deben adquirir durante sus estudios en el conservatorio superior una formación en materias educativas para poder desempeñar adecuadamente sus funciones, formación que no debería dejar al margen las TIC. En estos centros, el departamento de Pedagogía se encarga de todas las asignaturas relacionadas con lo educativo. De forma directa o indirecta, el profesorado es responsable del desarrollo de actitudes y concepciones de sus alumnos relativas a la educación y, también, con respecto al uso de las tecnologías en los procesos de enseñanza-aprendizaje. El propósito de este estudio, por lo tanto, es indagar acerca del uso que hacen de las TIC los docentes de la especialidad de Pedagogía en el proceso de enseñanza-aprendizaje y conocer su punto de vista en cuanto a las TIC para la ejercer la docencia en conservatorios.

\section{ESTADO DEL ARTE}

\subsection{Aportaciones de las TIC a la educación musical}

Diversos autores (Tejada, 2004; Savage, 2007; Masdéu, 2015; Rosas, Rocha y Behar, 2016) señalan efectos positivos que las TIC pueden ejercer cuando se incorporan adecuadamente en los procesos de enseñanza-aprendizaje de la música:

- Aumentar el tiempo de los procesos de instrucción musical, ya que pueden continuar fuera del aula. 
- Favorecer la comprensión de conceptos teóricos, el desarrollo de destrezas auditivas y la transferencia simbólica del sonido a la imagen mental.

- Motivar e involucrar al alumnado en su propia formación, ya que fomentan el aprendizaje activo, la exploración por parte del alumnado y la construcción del conocimiento.

- Flexibilizar e individualizar el ritmo de aprendizaje de los alumnos.

- Ampliar las posibilidades de creación de materiales didácticos.

- Fomentar la imaginación y la creatividad musical del alumnado.

- Ayudar al desarrollo de tareas cooperativas en el aula y fuera de ella.

A pesar de ello, en los conservatorios de música, el uso de las TIC - y, en general, la apuesta por la innovación educativa-, no es todavía algo generalizado. De hecho, en lo que respecta a la asignatura de Lenguaje Musical, aunque es una de las más importantes porque aglutina toda la base de contenidos teórico-prácticos, destrezas y aptitudes necesarias para una formación musical integral, es percibida por gran parte del alumnado como poco interesante y motivadora (Ordoñana, Laucirica y Tejada, 2004; Berrón, Balsera y Monreal, 2017). Uno de los factores que pueden estar contribuyendo a esta situación es que las prácticas pedagógicas en gran parte de los conservatorios y escuelas de música siguen ancladas en modelos tradicionales de enseñanza (Bautista y Fernández-Morante, 2018), ajenos a las investigaciones que promueven la mejora de la calidad educativa.

Sin embargo, en este ámbito existen investigaciones relacionadas con la implementación de determinadas tecnologías que plasman resultados positivos en el aprendizaje tanto en los niveles elementales y profesionales (Balo, Lago y Ponce de León, 2014; Bernabé y Azorín, 2015; Galera y Tejada, 2010; Ordoñana, Laucirica y Tejada, 2004) como en el superior (Timiraos, 2015). Asimismo, también existen algunos estudios más amplios relacionados con el uso de las TIC en estos centros (Belló, 2011; De la Rosa, 2015; Roig y Moncunill, 2012). No obstante, estas investigaciones también ponen de manifiesto que los docentes demandan una mejor formación en este ámbito, dado que en su mayoría no se consideran preparados para emplear adecuadamente las TIC en su labor pedagógica.

\subsection{El uso de las TIC por parte del profesorado: perspectivas y necesidades}

Gutiérrez, Palacios y Torrego (2010) defienden que, para una adecuada integración curricular de las TIC, se debería incidir en este aspecto desde la etapa de formación inicial de los futuros docentes. Sin embargo, estos autores también consideran que esta depende de la propia formación del profesorado que les prepara para la docencia. Sobre esta cuestión, Silva y Astudillo (2012) apuntan que en las facultades no existe una visión clara de cómo integrar las TIC en las propias prácticas pedagógicas del profesorado. Se tiende a utilizar el modelo instruccional tradicional, lo que dificulta que los alumnos puedan integrar las TIC en sus futuras clases como docentes. Cabe señalar que, en el ámbito de las enseñanzas superiores de música, son todavía escasos los estudios que analizan la presencia de las TIC en los centros y su uso por parte del profesorado (Belló, 2011; De la Rosa, 2015; Timiraos, 2015), y ninguno de ellos se centra en la formación inicial docente.

En línea con lo anterior, Cabero y Marín (2014) coinciden en que el uso que hace el profesorado de las TIC no suele ser de innovación educativa, si no que se articula alrededor de la motivación, comunicación y la transmisión de información. Una de las causas es la falta de una adecuada formación pedagógico-didáctica en relación con las tecnologías, más allá del conocimiento y dominio de herramientas informáticas (Hernández y Quintero, 2009; Herrada y Herrada, 2011). Las políticas educativas relacionadas con la formación permanente del profesorado también han sido sometidas a juicio en algunas investigaciones que revelan más un cumplimiento de requisitos y de formación técnica que de verdadera motivación por transformar la educación (Gewerc y Montero, 2011; Sánchez, Ramos y Sánchez, 2014). 


\section{MÉTODO}

Para llevar a cabo este estudio hemos optado por una metodología mixta que combina los resultados de un cuestionario y los de un grupo de discusión. En su enfoque predomina el componente interpretativo, dado que nuestra intención es examinar una realidad relativamente desconocida -apenas hay estudios que implican a este tipo de profesorado- para captar una perspectiva general de la misma y reflexionar sobre ella.

\subsection{Objetivos}

- Indagar el uso que hacen de las TIC los profesores de Pedagogía musical en los procesos de enseñanza-aprendizaje.

- Conocer el punto de vista del profesorado en cuanto a las TIC para la docencia en conservatorios.

\subsection{Participantes}

En el cuestionario participan los profesores de Pedagogía de los conservatorios superiores españoles. Esta especialidad se imparte en 14 centros públicos repartidos en 11 comunidades autónomas: Andalucía, Asturias, Baleares, Canarias, Cataluña, Comunidad Valenciana, Galicia, Madrid, Murcia, Navarra y País Vasco. Dada la naturaleza de estos centros, que cuentan con ratios bajas de alumnos, en ciertos casos no existe un departamento de Pedagogía exclusivo, sino que es compartido por otras especialidades como Musicología, Música Antigua o Canto. Así pues, el número de profesores que imparten asignaturas específicas de Pedagogía oscila entre 1 y 14 según el centro. Por otro lado, algunos de ellos imparten asignaturas que, en principio, pueden ser menos propensas al uso de las TIC. La estimación de profesores que puede contestar al cuestionario es de unos 40 si se toman en cuenta los datos sobre los miembros de departamentos que aparecen en las webs. A pesar de que no se trata de una muestra muy numerosa de docentes, su colaboración resulta valiosa para la investigación.

Por otro lado, para obtener más información y ahondar en el problema de una forma directa, se realiza un grupo de discusión con personas del ámbito educativo musical ubicadas en la Región de Murcia. En la sesión intervienen cuatro docentes de perfiles distintos: dos miembros del departamento de Pedagogía del conservatorio superior de Murcia, un miembro del departamento de Composición del mismo centro y un profesor de Lenguaje Musical del conservatorio profesional de la ciudad que ha cursado la especialidad de Pedagogía. De los docentes del conservatorio superior, dos son miembros del equipo directivo.

\subsection{Técnicas e instrumentos}

\subsubsection{Cuestionario}

Dado que no existe un cuestionario de las características que requiere el estudio, hemos construido uno a partir de otros instrumentos validados de diversas tesis doctorales que poseen cierta relación con la investigación, tras una búsqueda en torno a los siguientes temas: (a) las TIC en la enseñanza musical, (b) las TIC en la docencia universitaria, (c) la formación inicial del profesorado en TIC, (d) competencia digital docente. La selección de los ítems se basa en la adecuación de los mismos a los objetivos que persigue la investigación. En la Tabla 1 se muestra la relación entre los distintos bloques y las fuentes de las que se han tomado las preguntas.

Tabla 1. Bloques de preguntas del cuestionario

\begin{tabular}{lcl}
\hline \multicolumn{1}{c}{ Bloque } & Ítems & \multicolumn{1}{c}{ Fuentes } \\
\hline 1. Datos personales & $1-6$ & $\begin{array}{l}\text { De la Rosa (2015), González (2017), } \\
\text { Henríquez (2002), Masdéu (2015) }\end{array}$ \\
2. Características del centro y del aula & & $\begin{array}{l}\text { De la Rosa (2015), González (2017), } \\
\text { Román (2015) }\end{array}$
\end{tabular}


3. Formación TIC del docente

4. Las TIC en la docencia y en la formación de docentes

5. Creencias y valoraciones sobre las TIC en la educación
$13-23$

$24-43$

$34-40$
Arques (2013), Carrillo (2014), De la Rosa (2015), Gutiérrez (2011), Masdéu (2015), Román (2015)

De la Rosa (2015), Echeverría (2011), Henríquez (2002), Masdéu (2015), Salinas (2014)

Gutiérrez (2011), Henríquez (2002), González (2017)

El instrumento resultante consta de cinco bloques que reúnen un total 40 ítems, tanto de respuesta cerrada como de respuesta abierta. En lo que respecta a la redacción final de los mismos, se han mantenido literales en lo posible, así como la tipología de respuesta asociada. En algunos casos se ha modificado ligeramente la expresión lingüística y las opciones de respuesta con el objetivo de dotar al cuestionario de mayor coherencia; también se han combinado opciones de respuesta de algunas fuentes que planteaban cuestiones semejantes. Por último, dos de los ítems se han formulado de manera original como consecuencia de no haber hallado cuestionarios directamente relacionados con la utilización de las TIC en los niveles elementales y profesionales de música.

Finalmente, enviamos un e-mail de invitación a la jefatura de estudios de los diferentes centros para su distribución entre el profesorado de la especialidad a través de un enlace de Google Forms. De las 40 respuestas estimadas recibimos finalmente un total de 24 que varían entre 1 y 4 según el centro. Quedan sin responder 2 de los 14 departamentos invitados.

\subsubsection{Grupo de discusión}

Mediante esta técnica perseguimos una doble finalidad: por un lado, recoger más datos para enriquecer y contrastar la información obtenida de los cuestionarios y, por otro, promover un debate en torno a las cuestiones tratadas en la investigación. Los temas propuestos se plantean en forma de preguntas antecedidas, en algunos casos, por una breve contextualización. No obstante, representan un guion flexible que se adapta a la dinámica generada:

- ¿De qué manera integráis las tecnologías en vuestras clases?

- Algunos profesores del centro utilizan sistemas de gestión del aprendizaje. ¿Se trata de iniciativas personales, se propicia el uso de estas plataformas?

- ¿Existe intención de incrementar y normalizar el uso de las TIC? ¿Se respiran aires de cambio?

- ¿Qué papel pueden tener las TIC en la enseñanza de Lenguaje Musical?

- ¿Qué tipo de formación necesita el profesorado del departamento de Pedagogía para su labor docente, en relación con las TIC?

- Según los resultados de los cuestionarios, la mayoría profesorado afirma que su conocimiento acerca de las posibilidades de las TIC en cuanto a la formación de los alumnos es "regular". ¿Qué influencia puede tener esto en la formación de futuros pedagogos?

- La mayoría de encuestados señala que su formación en TIC es informal, es decir, autodidacta o a partir de información compartida con compañeros de trabajo. Por debajo del $50 \%$ se habla de distintos tipos de formación específica. ¿Cómo interpretáis estos datos?

La sesión se graba en audio, previo consentimiento de todos los miembros, y dura alrededor de 1 hora y 20 minutos. Se desarrolla en un ambiente cómodo y tranquilo; los participantes se expresan libremente e interactúan a menudo entre ellos, aportando su visión de cada tema y comentando ideas que surgen de la escucha atenta entre unos y otros. Posteriormente, los datos se transcriben para poder leerlos e identificar temas, conceptos y puntos que coincidan con los datos anteriores de la investigación, así como la posible aparición de ideas emergentes que aporten interés a la investigación o a futuras líneas de trabajo. 


\section{RESULTADOS}

\subsection{Resultados del análisis de los cuestionarios}

\subsubsection{Datos personales}

La distribución por sexos del profesorado es de 16 de mujeres y 8 hombres, es decir, un $66,7 \%$ y un $33,3 \%$ respectivamente. Las edades superan los 30 años en todos los casos, y es más común el profesorado que supera los 50 (58,3\%). La mayoría de departamentos ha tenido representación en el cuestionario, como muestra la Tabla 2.

Tabla 2. Distribución de participantes en el cuestionario

\begin{tabular}{lcl}
\hline \multicolumn{1}{c}{ Centro } & Respuestas \\
\hline Conservatorio Superior de Música de A Coruña & 1 \\
Conservatorio Superior de Música de Alicante & 1 \\
Conservatorio Superior de Música de las Islas Baleares & 1 \\
Conservatorio Superior de Música de Canarias & 4 \\
Conservatorio Superior de Música de Castellón & 2 \\
Conservatorio Superior de Música de Murcia & 3 \\
Conservatorio Superior de Música de Navarra & 1 \\
Conservatorio Superior de Música de Oviedo & 0 \\
Conservatorio Superior de Música de Valencia & 0 \\
Conservatorio Superior de Música de Vigo & 1 \\
ESMUC (Escola Superior de Música de Catalunya) & 2 \\
Musikene (Centro Superior de Música del País Vasco) & 4 \\
Real Conservatorio Superior de Música de Granada & 2 \\
Real Conservatorio Superior de Música de Madrid & 2 \\
TOTAL & 24 \\
\hline
\end{tabular}

En cuanto a la titulación académica, la mayoría (37,5\%) posee únicamente estudios superiores de música, mientras que el 29,2\% combina esta titulación con estudios universitarios y otro $29,2 \%$ tiene un doctorado (Figura 1). Por otro lado, se puede afirmar que la mayor parte del profesorado $(70,8 \%)$ posee una experiencia docente de 20 años o más.
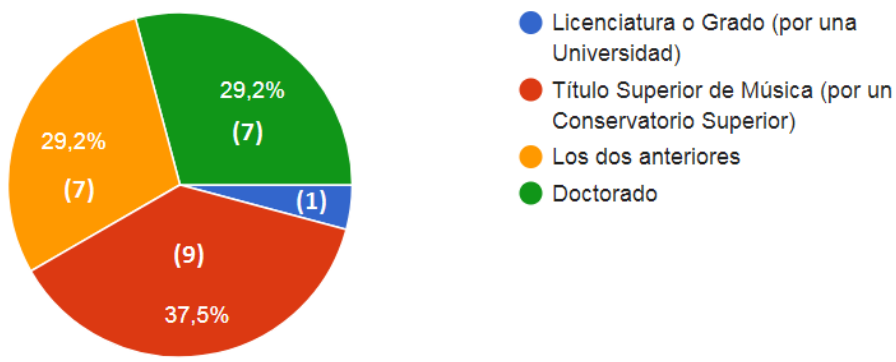

Figura 1. Tipo de titulación académica

\subsubsection{Características del centro y del aula}

Una amplia mayoría de encuestados afirma que el centro cuenta con conexión a Internet en todo su espacio $(91,7 \%)$ y que, en el caso de su aula, el porcentaje asciende al 95,8\%. Es decir, 
al menos en teoría, la disponibilidad para trabajar conectados a la red está asegurada. El $78,3 \%$ del profesorado afirma utilizar muy a menudo Internet en el aula, mientras que un $21,7 \%$ solo alguna vez (Figura 2).
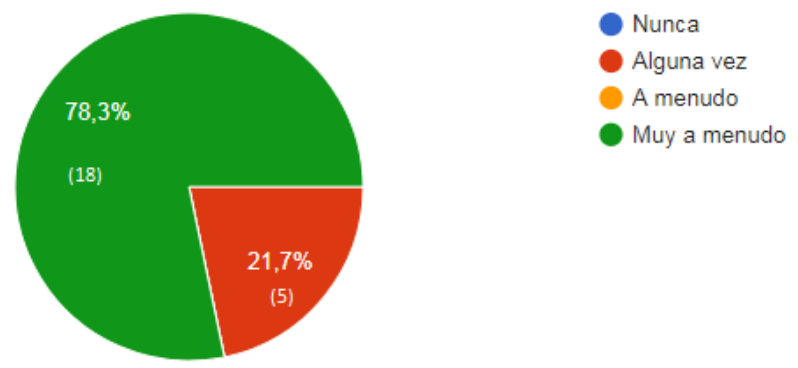

Figura 2. Frecuencia de uso de Internet en el aula

Sin embargo, la dotación de ordenador u ordenadores es en el aula no es tan alta, ya que el $20,8 \%$ indica la ausencia de este equipamiento. Del $79,2 \%$ restante, el $90 \%$ lo utiliza muy a menudo durante sus clases, mientras que $10 \%$ lo utiliza a menudo. Por otro lado, el $70,8 \%$ considera que el aula está preparada para trabajar con los alumnos usando las TIC mientras que el $29,2 \%$ considera que no.

\subsubsection{Formación del docente en TIC}

En general, el nivel que afirman tener los profesores es de medio $(25 \%)$ a experto $(20,8 \%)$, siendo más común el nivel avanzado (37,5\%). Solo un $16,7 \%$ considera que posee un nivel básico (Figura 3). Conviene aclarar que en los cuestionarios se establecieron una serie de indicadores para orientar al profesorado en los conocimientos de cada nivel.
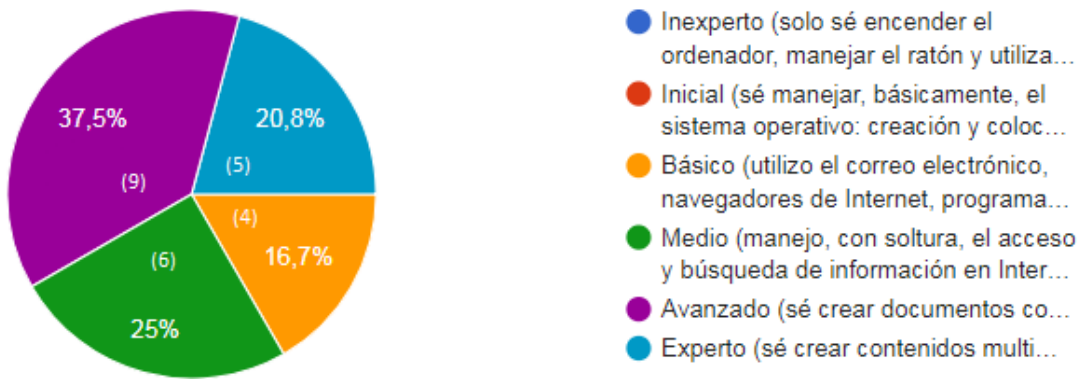

Figura 3. Nivel de uso de las TIC

En cuanto al nivel de conocimiento sobre las posibilidades de las TIC para enriquecer la práctica docente, el profesorado afirma que es regular $(41,7 \%)$, alto $(41,7 \%)$ y muy alto $(16,7 \%)$, por lo que en general la valoración es positiva y cercana al $58 \%$ si consideramos a partir de "alto". No ocurre así en el nivel de conocimiento del papel de las TIC en la formación del alumnado. En este caso es ligeramente menor, ya que más de la mitad del profesorado $(54,2 \%)$ considera que posee un conocimiento regular frente a los que lo consideran alto $(29,2 \%)$ o muy alto $(16,7 \%)$.

Con respecto a la formación recibida sobre las TIC en general, el 58,3\% responde que sí la ha obtenido frente al $41,7 \%$ que no, mientras que en el área de TIC para la educación musical el $50 \%$ del profesorado no ha recibido ninguna. En cuanto al tiempo invertido en cursos, de los 16 profesores que han respondido a esta pregunta, solo un tercio supera las 100 horas de formación (Figura 4). 


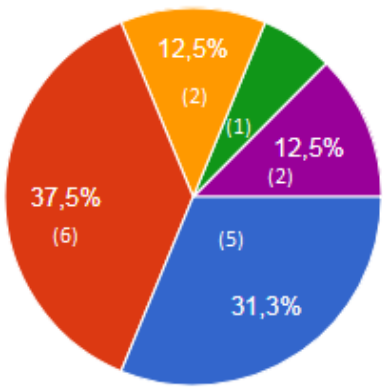

De 0 a 50 horas

De 51 a 100 horas

De 101 a 200 horas

De 201 a 300 horas

Más de 300 horas

Figura 4. Horas dedicadas a la formación en TIC

La mayoría de profesores afirman que han realizado una formación autodidacta $(95,8 \%)$ o compartiendo información con otros compañeros de trabajo (58,3\%). De hecho, este tipo de aprendizaje informal es el más común, seguido por el $45,8 \%$ de profesores que han realizado cursos de formación organizados por los centros de formación del profesorado. También se les ha preguntado acerca del tipo de acciones que realizan para mejorar su competencia con las TIC. La Figura 5 muestra la frecuencia con que realizan determinada acción. Cabe destacar que no es muy común la participación en espacios de reflexión o en grupos de innovación e investigación sobre la docencia con las TIC, ni tampoco la difusión de experiencias docentes relacionadas. En cambio, encuentran una mayor frecuencia el uso de fuentes de información diversas y el acceso a plataformas y repositorios de recursos digitales.
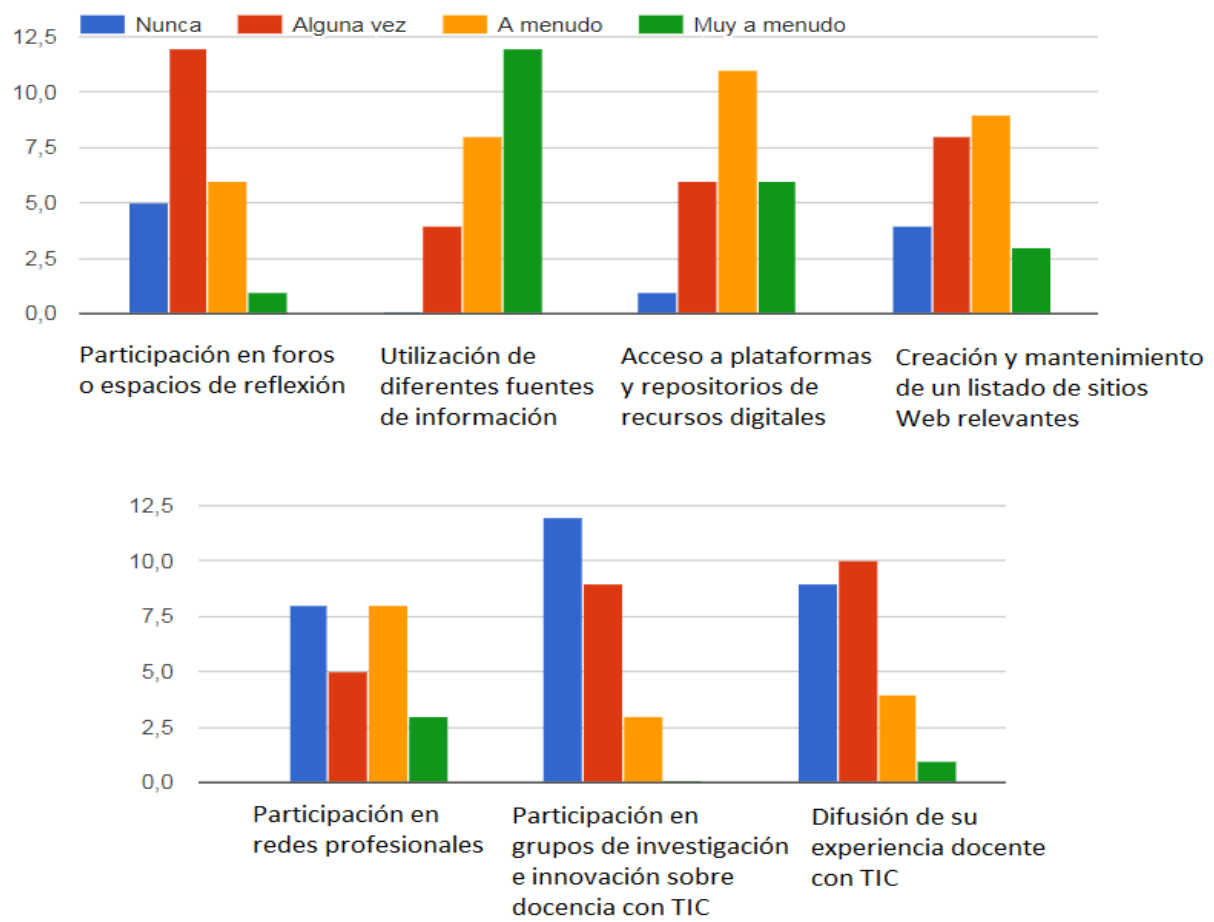

Figura 5. Acciones realizadas para mejorar la competencia en TIC

El $87,5 \%$ de los encuestados afirma necesitar mayor formación en TIC. El ítem 23 ofrece un espacio para responder libremente sobre las necesidades que cada docente considera que debería cubrir. Hemos categorizado las respuestas obtenidas y contado las frecuencias (Tabla 3). Para ello, hemos descompuesto aquellas respuestas que contenían varios elementos distintos en fragmentos más pequeños, procurando identificar unidades gramaticales que representaran adecuadamente las categorías. En general, se observa que la formación demandada es de tipo técnico. 
Tabla 3. Necesidades formativas del profesorado

\begin{tabular}{lc}
\hline \multicolumn{1}{c}{ Categoría } & Frecuencia \\
\hline Herramientas y software & 17 \\
Uso educativo de las TIC & 6 \\
Recursos para el trabajo y la investigación & 4 \\
\hline
\end{tabular}

\subsubsection{Las TIC en la docencia y en la formación de docentes}

Todos los encuestados utilizan las TIC de una u otra manera en su labor docente. Los usos más destacados (Figura 6) son la audición de música $(91,7 \%)$, la preparación de materiales $(91,7 \%)$ y la exposición de presentaciones $(91,7 \%)$, seguido de la proyección de vídeos $(87,5 \%)$, la comunicación $(70,8 \%$ y la realización de actividades interactivas. De forma puntual, algunos profesores han señalado otros usos como la evaluación del alumnado, la gestión del espacio virtual de aprendizaje u otros usos de creatividad musical y audiovisual.

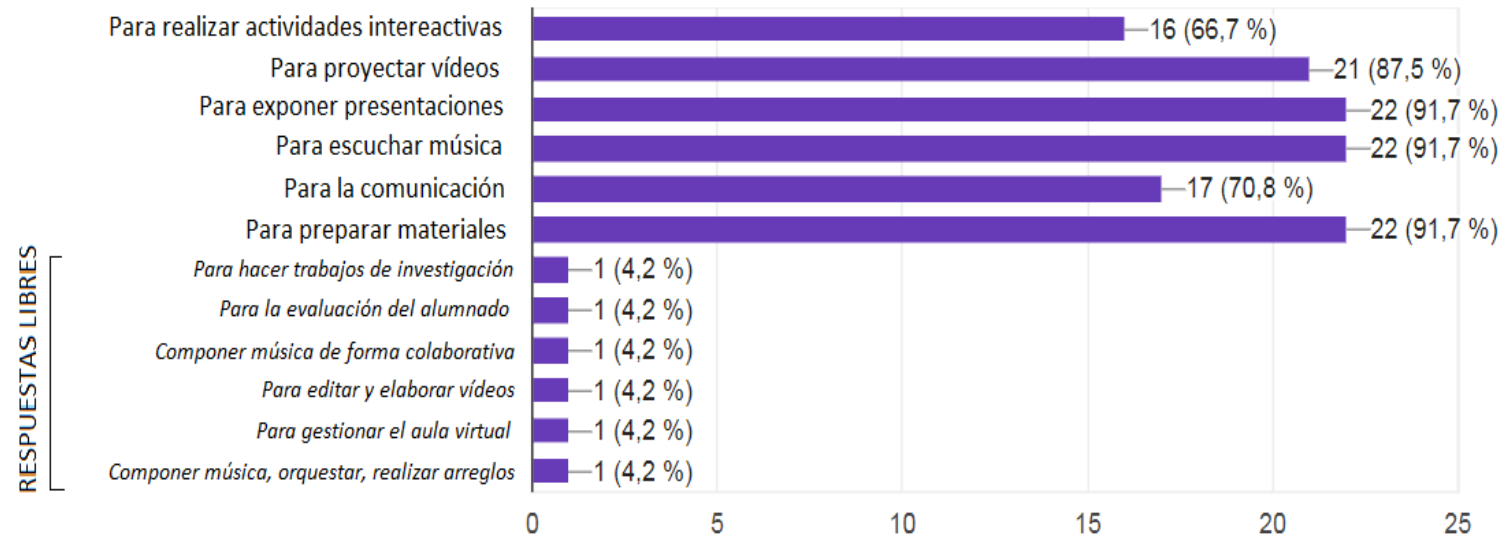

Figura 6. Usos de las TIC en la práctica docente.

En cuanto a los dispositivos y recursos generales más utilizados, encabezan la lista el ordenador (100\%), el equipo de audio (95,8\%), e Internet para realizar búsquedas $(95,8 \%)$, seguido del videoproyector $(79,2 \%)$, las presentaciones $(75 \%)$ y las páginas web educativas $(70,8 \%)$. En menor medida, entre el $40 \%$ y el $60 \%$ utiliza aplicaciones ofimáticas, aplicaciones educativas, plataformas educativas como Moodle o Schoology, redes sociales y blogs. Por debajo se sitúa el uso de foros $(37,5 \%)$, portafolio de aula $(20,8 \%)$ y pizarra digital interactiva $(25 \%)$. La frecuencia con la que el profesorado hace uso de las TIC en la práctica docente es alta, puesto que un $62,5 \%$ las utiliza muy a menudo y un $20,8 \%$ indica hacerlo a menudo.
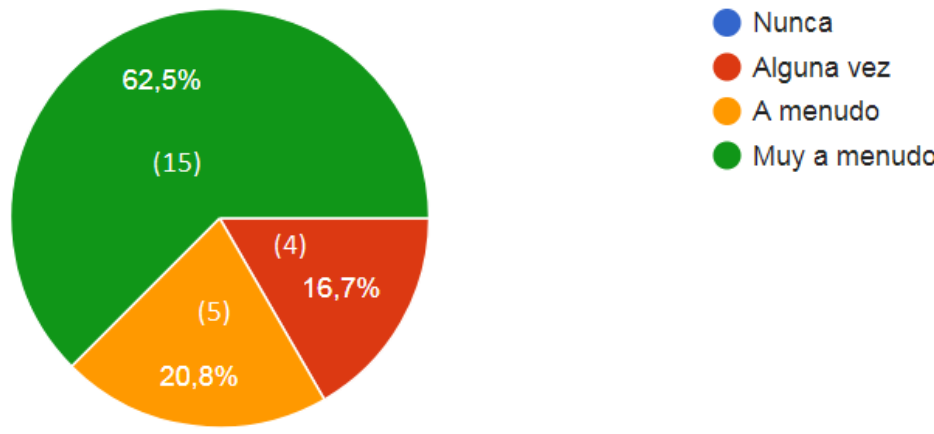

Figura 7. Frecuencia de uso de las TIC en la práctica docente 
Los objetivos con que se utilizan las TIC son muy diversos. Por este motivo, los agrupamos dos intervalos: del $70 \%$ al 90\% utilizan las TIC para la ampliación de información, clarificar e ilustrar conceptos, desarrollar actividades de práctica y motivar al alumnado. Entre el $50 \%$ y el $70 \%$ señalan el fomento del autoaprendizaje, apoyar el desarrollo de las lecciones o promover el trabajo en equipo. Con respecto al tipo de actividades que los alumnos desarrollan con las TIC, la mayoría de docentes señalan la búsqueda de información (87,5\%), la comunicación con profesores y compañeros $(87,5 \%)$ o la realización de trabajos con procesadores de texto $(83,3 \%)$ $\mathrm{u}$ otras herramientas multimedia $(70,8 \%)$.

\subsubsection{Creencias y valoraciones sobre las TIC en la educación}

El $100 \%$ del profesorado considera que las TIC pueden ser un recurso importante para mejorar la enseñanza. En cuanto a las características más valoradas, el 83,3\% señala la interactividad y, en la misma medida, la multivariedad de código de información. El 66,7\% indican que facilita el aprendizaje autónomo y un $62,5 \%$ destaca la motivación. Para filtrar un poco más estas creencias, se les solicita que marquen las tres posibilidades que consideren más significativas de las TIC en el proceso de enseñanza-aprendizaje. Las más votadas son el acceso a la información $(79,2 \%)$, la flexibilización de tiempos $(66,7 \%)$ y la comunicación interpersonal (54,2\%), como se observa en la Figura 10.

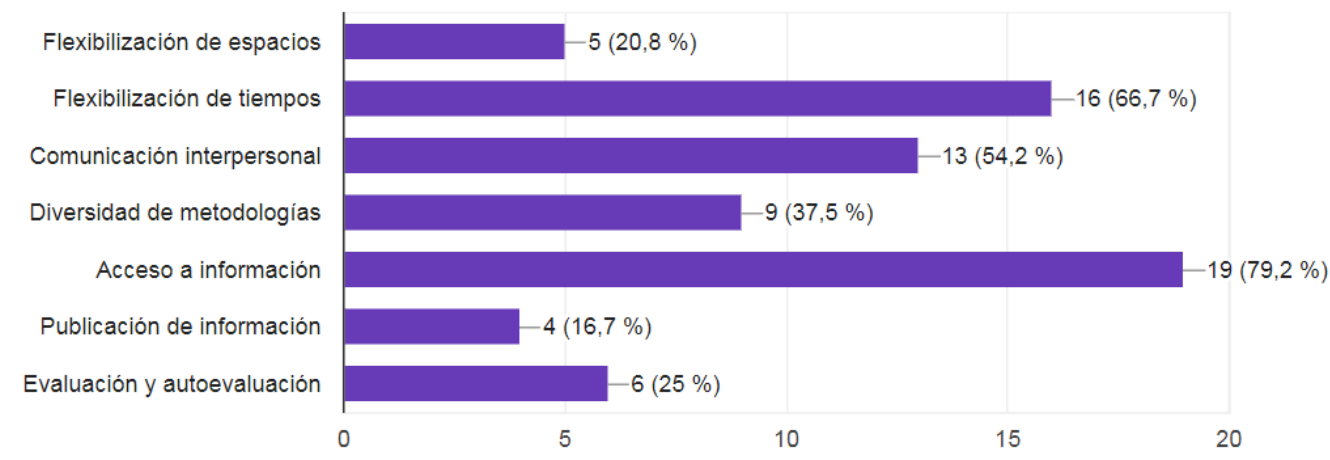

Figura 10. Posibilidades más significativas de las TIC

Sobre la integración de las TIC en las enseñanzas elementales y profesionales de música, el $79,2 \%$ del profesorado presenta un grado de acuerdo alto y el $20,8 \%$ un grado intermedio. Concretamente, en la enseñanza de la asignatura Lenguaje Musical, el profesorado responde abiertamente acerca de las ventajas que las TIC pueden aportar. En la Tabla 4 se muestran las respuestas filtradas y agrupadas en 7 categorías con sus frecuencias. Las más repetidas son la motivación del alumnado, la adaptación y flexibilización del proceso de enseñanza-aprendizaje y la autonomía en el estudio. Esto es muy significativo dado que la asignatura de Lenguaje musical suele presentar problemas relacionados con la falta de interés y con la dificultad para adquirir los conocimientos por parte del alumnado.

Tabla 4. Respuestas sobre el beneficio de las TIC en Lenguaje Musical

\begin{tabular}{lc}
\hline \multicolumn{1}{c}{ Categoría } & Frecuencia \\
\hline Motivación del alumnado & 8 \\
Adaptación y flexibilización del proceso de E-A & 8 \\
Autonomía en el estudio & 7 \\
Diversidad de recursos y de metodologías & 6 \\
Entrenamiento de destrezas & 4 \\
Creatividad & 3 \\
Dinamización del aprendizaje & 3 \\
\hline
\end{tabular}


Hemos realizado el mismo proceso a propósito de los beneficios de las TIC en la enseñanza del instrumento (Tabla 5). El principal uso de las TIC es el acceso de vídeos para visualizar versiones diferentes de una interpretación, observar posturas corporales, conocer técnicas de ejecución o ver masterclasses, entre otros ejemplos. Por otro lado, también destaca la utilización de las tecnologías para orientar al alumnado en la gestión de su aprendizaje, además del acceso a la información. Otro uso destacable es el empleo de grabaciones y portafolios para propiciar el seguimiento y la evaluación del alumno y para que éste realice su propia autoevaluación.

Tabla 5. Respuestas sobre el beneficio de las TIC en la enseñanza del instrumento

\begin{tabular}{lc}
\hline \multicolumn{1}{c}{ Categoría } & Frecuencia \\
\hline Vídeos y audiciones con distintos fines & 11 \\
Autonomía en el aprendizaje & 8 \\
Acceso a información & 7 \\
Herramienta para el seguimiento, evaluación y & 5 \\
autoevaluación & \\
Motivación & 5 \\
Acceso a partituras y software auxiliar & 3 \\
Creatividad & 2 \\
Comunicación & 2 \\
\hline
\end{tabular}

\subsection{Resultado del análisis del grupo de discusión}

Después de realizar la transcripción completa, leemos atentamente el texto para organizar las ideas surgidas a lo largo de la sesión, que se desarrolla a partir del guion previsto. Por ello, exponemos las ideas que en ese orden y citamos textualmente algunas afirmaciones representativas.

\subsubsection{Integración de las TIC en el aula. Experiencias personales}

Todos los participantes muestran acuerdo en la mejoría que supone el uso de las TIC en sus respectivas parcelas de enseñanza. Los usos más destacados son la comunicación y utilización de diferentes vías para proporcionar materiales de clase y de refuerzo. También se comenta sobre el uso de aplicaciones que mejoran la autonomía del estudio en casa y programas de entrenamiento de destrezas auditivas. Sin embargo, se señala la importancia del trabajo presencial en el aula como parte insustituible de este tipo de aprendizaje.

P4. "El programa de entrenamiento auditivo lanza ejercicios, pero el trabajo previo que debe existir no está en el programa".

\subsubsection{Uso de sistemas de gestión del aprendizaje en el CSM de Murcia}

Su uso no es común, sino que deriva de iniciativas personales, pero por diferentes motivos, según debaten los participantes. Por un lado, se percibe mucha resistencia por parte del profesorado a utilizar las nuevas tecnologías. Esta idea se justifica, en parte, porque algunas plataformas tienen una usabilidad compleja y se requieren conocimientos informáticos que muchos profesores no tienen. Se comenta que muchas veces las Administraciones no dotan a los centros de un soporte común para todos los profesores, con lo que cada uno utiliza la herramienta o la manera que le resulta más adecuada para gestionar sus clases.

\subsubsection{Integración de las TIC en los conservatorios}

La cuestión relativa a la dotación de medios por parte de la Administración lleva al debate acerca del uso de las TIC en los centros. Los participantes comentan que, al tratarse de centros diferentes a los de régimen general, a veces no existen recursos adecuados. Otro de los motivos que se aducen es que, en el caso de los conservatorios superiores, en muchas ocasiones los profesores no sienten la necesidad de cambiar de prácticas o de buscar recursos más 
motivadores, porque sus alumnos ya acuden al centro motivados ya que se profesionalizan en lo que les gusta. Sin embargo, los alumnos de los conservatorios elementales y profesionales no tienen siempre esta motivación. Los participantes expresan acuerdo sobe que, en los conservatorios, existe generalmente poco interés en cambiar de metodologías de enseñanza.

$\mathrm{P} 1$. "Nos escudamos en que la música se ha enseñado siempre de una determinada forma [...] y parece ser que no cuaja la idea de que hay recursos que te pueden auxiliar en ese proceso".

\subsubsection{Problemas de la asignatura de Lenguaje Musical. Papel de las TIC en su docencia}

Se discute acerca de lo ambicioso que es el currículo de enseñanzas elementales y profesionales para el poco tiempo de trabajo que hay en el aula. Otra cuestión es que no se trabaja adecuadamente el desarrollo de destrezas y competencias para que el alumno sea autosuficiente en su estudio. Y es aquí donde pueden jugar un gran papel las TIC.

$P 1$. "Cuando el niño llega a su casa y se le dice que estudie, no sabe qué hacer. Sabe que tiene que aprender una lección, pero no tiene las pautas necesarias para abordarla. Las TIC pueden servir parar pautar esas cosas que el niño tiene que hacer en casa".

Para tal fin se menciona el enfoque del aula invertida o flipped classroom. Mediante una serie de vídeos, el alumno puede tener al profesor en casa y saber cómo abordar un ejercicio de cualquier tipo. No obstante, se presenta el inconveniente de que hay que preparar recursos específicos para ello, ya que a veces los que hay publicados no se adaptan a las clases del conservatorio, por lo que el profesorado tiene que consumir mucho tiempo y esfuerzo para elaborarlos. Además, muchos centros no cuentan con recursos tecnológicos en sus aulas: la dotación habitual es la pizarra tradicional y el piano. De hecho, en los centros que cuentan con pizarra digital y otros recursos TIC, en general hay un gran desconocimiento sobre cómo utilizarlos, por lo que se termina haciendo con ellos lo mismo que se venía haciendo antes.

P3. "La pizarra digital...la usas como la pizarra de tiza, pero sin mancharte las manos".

Sin embargo, hay un acuerdo general en que lo importante no es que un profesor sepa utilizar las TIC, sino que tenga inquietud para innovar desde el punto de vista metodológico. Es decir, es una cuestión de actitud ante la enseñanza. Se aprende a utilizar diversas herramientas pero no hay una incidencia real en la manera en que esto puede cambiar la educación en las aulas. Es decir, que debería estimularse un cambio de pensamiento pedagógico de base, ya que las tecnologías por sí solas no van a cambiar nada.

\subsubsection{Formación en TIC del profesorado de Pedagogía}

Para abordar esta cuestión se contextualiza a los participantes a través de datos extraídos de los cuestionarios: el nivel de formación es alto, pero sobre todo en lo que se refiere a usos técnicos para el desempeño de labores docentes. Sin embargo, en lo que respecta a los usos didácticos de las TIC o las implicaciones de las mismas en la formación del alumnado, el nivel de conocimiento es menor.

Una de las causas podría encontrarse en los cursos que puede haber recibido este profesorado se centran más en el manejo instrumental y no tanto en el aspecto pedagógico. $Y$ esta carencia de formación en usos didácticos, en el cambio de enfoque de la enseñanza, no predispone al profesorado a ninguna innovación significativa. Esto sucede también en el ámbito de las enseñanzas profesionales y elementales. Así, la innovación sólo se da en aquellos docentes que tengan una cierta iniciativa creadora, en aquellos que no tengan miedo a salirse de lo cómodo, de lo que ya se viene haciendo desde siempre por inercia. Sin embargo, este esfuerzo no está reconocido muchas veces ni por los centros ni por la Administración, según señalan los participantes.

\section{CONCLUSIONES Y DISCUSIÓN}

Hemos podido comprobar que el profesorado de Pedagogía utiliza recursos informáticos y tecnológicos en los procesos de enseñanza-aprendizaje con bastante frecuencia. Sin embargo, las TIC tienen, en general, un papel poco innovador en la formación de los alumnos, ya que se 
emplean como actualización o mejora de lo que ya se venía haciendo anteriormente: desarrollo de las lecciones con soportes audiovisuales o realización de ejercicios y tareas con distintos procesadores y editores, entre otros usos. En este sentido la investigación coincide con lo expuesto por Cabero y Marín (2014) y Silvia y Astudillo (2012) con respecto al enfoque tradicional de la docencia con las TIC. En el ámbito de los conservatorios de música, esta resistencia a buscar nuevos enfoques didácticos es todavía más acusada, tal y como han confirmado los participantes del grupo de discusión.

Este hecho podría relacionarse con que más de la mitad de docentes encuestados considera que su conocimiento del papel de las TIC en la formación del alumnado es regular, independientemente de que la mayoría utilizan recursos tecnológicos con frecuencia. A pesar de que los centros cuentan con un equipamiento suficiente, existe todavía bastante desconocimiento acerca cómo utilizarlos de una manera novedosa. Por otro lado, esto también se puede asociar a la escasa actividad del profesorado en relación con la investigación e innovación sobre la docencia con las TIC. Sería conveniente incentivar la actividad investigadora de los docentes desde los propios centros y desde las Administraciones, además de ofertar formación en didáctica con las TIC. Es preciso fijar metas y avanzar de forma colectiva para que el esfuerzo produzca resultados significativos. Por otro lado, que las Administraciones reconozcan la labor del profesorado que innova puede ser otra de las claves que para que tenga lugar un cambio generalizado en la realidad educativa.

Todas las personas que han colaborado en la investigación coinciden en que las TIC suponen un recurso valioso en el proceso de enseñanza-aprendizaje. Evidentemente, para poder integrarlas de forma sistemática es necesaria una adecuada formación del profesorado en TIC y que exista una dotación mínima en equipamientos. Pero esto se debería abordar no solo desde el aspecto tecnológico, sino desde el plano pedagógico que ha de sustentar el cambio real. Podría decirse que es el deber de los profesores que forman a los futuros docentes el incentivar la búsqueda de nuevas formas de enseñar y normalizar un uso reflexivo de las tecnologías. En el caso de los conservatorios, esto resulta urgente teniendo en cuenta lo difícil que resulta cuesta cambiar las prácticas educativas perpetuadas y arraigadas en la costumbre.

\section{ENLACES}

Cuestionario:

https://docs.google.com/forms/d/e/1FAlpQLSeMLC5wMklttXS7N5F6n1QoLuh0bhk1pJOxatU8

W3mbp zAcw/viewform

\section{REFERENCIAS BIBLIOGRÁFICAS}

Area, M. (2007). Algunos principios para el desarrollo de buenas prácticas pedagógicas con las TICs en el aula. Comunicación y pedagogía: Nuevas tecnologías y recursos didácticos, 222, 42-47.

Arques, R. (2013). Las actitudes del profesorado de música ante las Tecnologías de la Información y la Comunicación. (Tesis doctoral) Universidad de Alicante, Alicante.

Balo, M., Lago, P. y Ponce de León, L. (2014). Los alumnos ante el dictado musical. Las TIC como aliadas para mejorar las experiencias. Didáctica, Innovación y Multimedia, 28, 1-14. Recuperado de: https://ddd.uab.cat/record/131726

Bautista, A. y Fernández-Morante, B. (2018). Monográfico sobre Investigación en Interpretación Musical: Implicaciones para el Desarrollo Profesional Docente. Psychology, Society, \& Education, 10 (1), 1-13. DOI: http://dx.doi.org/10.25115/psye.v10i1.1869

Belló, J. (2011). Las tecnologías de la Información y Comunicación en los conservatorios de música de Galicia. Análisis de los usos, actitudes y formación del profesorado. Tesis doctoral. Universidad de A Coruña, A Coruña. 
Bernabé, M., y Azorín, J. (2015). La pizarra digital en la clase de lenguaje musical: aprendizaje armónico-analítico. Opción, 31, 235-249. Recuperado de: http://www.redalyc.org/html/310/31045567013/

Berrón, E., Balsera, F.J. y Monreal, I.M. (2017). Ansiedad de los alumnos en la asignatura de Lenguaje Musical y estrategias para reducirla. Revista Internacional de Educación Musical, 5, 41-48. DOI: 10.12967/ RIEM-2017-5- p041-048

Cabero, J. y Marín, V. (2014). Miradas sobre la formación del profesorado en tecnologías de información y comunicación (TIC). Enl@ce Revista venezolana de Información, Tecnología y Conocimiento, 11 (2), 11-24. Recuperado de: http://www.redalyc.org/articulo.oa?id=82332625005

Carrillo, D. B. (2014). Competencias TIC de los docentes para la enseñanza mediante entornos virtuales en educación superior. El caso de la Universidad de lo Andes-Venezuela: evaluación y diseño de un plan de formación. (Tesis doctoral). Universitat Rovira i Virgili, Tarragona. Recuperado de: https://www.tdx.cat/handle/10803/285330

De la Rosa, E. (2015). El uso de las TIC en los centros superiores de música. Un análisis desde la perspectiva del profesorado, del alumnado y de los recursos de las webs institucionales. (Tesis doctoral) Universidad de las Palmas de Gran Canaria, Las Palmas de Gran Canaria.

Echeverría, A. C. (2011). TICs en la formación inicial y permanente del profesorado de educación especial: Universidad de Costa Rica. (Tesis doctoral) Universidad Complutense de Madrid, Madrid. Recuperado de: http://redined.mecd.gob.es/xmlui/handle/11162/102174

Galera, M. y Tejada, J. (2010). Editores de partitura y procesos implicados en la lectura musical. Lista Electrónica Europea de Música en la Educación, 25, 65-75. Recuperado de: http://musica.rediris.es/leeme/revista/galera\&tejada10.pdf

Gewerc, A. y Montero, L. (2011). Culturas, formación y desarrollo profesional. La integración de las TIC en las instituciones educativas. Revista de Educación, 362, 323-347. DOI: 104438/1988-592X-RE-2011-362-163

González, A. (2017). Análisis y evaluación de la competencia digital en la formación inicial del profesorado como elemento clave de mejora de la calidad educativa. (Tesis doctoral). Universidad Autónoma de Madrid, Madrid. Recuperado de: https://repositorio.uam.es/handle/10486/680253

Gutiérrez, A., Palacios, A. y Torrego, L. (2010). La formación de los futuros maestros y la integración de las TIC en la educación: anatomía de un desencuentro. Revista de Educación, 352, 267-293. Recuperado de: http://www.revistaeducacion.educacion.es/re352 TIC.html

Gutiérrez, I. (2011). Competencias del profesorado universitario en relación al uso de las Tecnologías de la Información y Comunicación: análisis de la situación en España y propuesta de un modelo de formación. (Tesis doctoral). Universitat Rovira i Virgili, Tarragona. Recuperado de: https://www.tesisenred.net/handle/10803/52835

Henríquez, M. A. (2002). Formación del profesorado en las Tecnologías de la Información y la Comunicación. Casos: ULA-URV. (Tesis doctoral). Universitat Rovira i Virgili, Tarragona. Recuperado de: https://www.tesisenred.net/handle/10803/8904

Hernández, M. y Quintero, A. (2009). La integración de las TIC en el currículo: necesidades formativas e interés del profesorado. Revista Electrónica Interuniversitaria de Formación del Profesorado, 12 (2), 103-119. Recuperado de: https://www.aufop.com/aufop/uploaded files/articulos/1248479648.pdf

Herrada, R. y Herrada, G. (2011). Adaptación de los estudios de magisterio al EEES: Las TIC en los nuevos planes de estudio. Edutec, 36. Recuperado de: http://www.edutec.es/revista/index.php/edutec-e/article/view/405/141

Masdéu, E. (2015). The digitalisation of music classrooms in schools in Catalonia: study of and proposal for a general framework for integrating technology in music education. (Tesis 
doctoral). Universitat de Lleida, Lleida. Recuperado de: https://www.tesisenred.net/handle/10803/300305

Ordoñana, J.A., Laucirica, A. y Tejada, J. (2004). Estudio cualitativo sobre el uso de programas informáticos para el desarrollo de destrezas rítmicas en la enseñanza musical especializada. Revista de psicodidáctica, 17, 127-136. Recuperado de: http://www.ehu.eus/ojs/index.php/psicodidactica/article/view/182/178

Roig, R. y Moncunill, M. (2012). Las TIC y el profesorado en el contexto educativo musical: un estudio en el Conservatorio Profesional de Música de Alicante. Comunicación presentada en el I Congreso Virtual Internacional sobre Innovación Pedagógica y Praxis Educativa, Sevilla, España.

Román, M. (2014). Las TIC en la educación musical en los centros de Educación Primaria de la Comunidad de Madrid: formación y recursos del especialista de música. (Tesis doctoral). Universidad Autónoma de Madrid, Madrid. Recuperado de: https://repositorio.uam.es/handle/10486/660734

Rosas, F.W., Rocha, L. y Behar, P.A. (2016). Music technology competencies for education: a proposal for a pedagogical architecture for distance learning. Actas del 13th International Conference on Cognition and Exploratory Learning in Digital Age (CELDA), 77-85.

Sánchez, P., Ramos, J. y Sánchez, J. (2014). Formación continua y competencia digital docente: el caso de la Comunidad de Madrid. Revista Iberoamericana de Educación, 65, 91-110. Recuperado de https://rieoei.org/RIE/article/view/395

Savage, J. (2007). Reconstructing music education through ICT. Research in Education, 78(1), 65-77.

Serrano, R.M. (2017). Tecnología y educación musical obligatoria en España: referentes para la implementación de buenas prácticas. Revista Electrónica Complutense de Investigación en Educación Musical, 14, 153-169. DOI: http://dx.doi.org/10.5209/RECIEM.54848

Silva, J. y Astudillo, A. (2012). Inserción de TIC en la formación inicial docente: barreras y oportunidades. Revista Iberoamericana de Educación, 58(4), 1-12. Recuperado de: https://rieoei.org/historico/deloslectores/4557Silva.pdf

Tejada, J. (2004). Música y mediación de la tecnología en sus procesos de aprendizaje. Educación XXI, 7, 15-26. DOI: https://doi.org/10.5944/educxx1.0.7.327

Timiraos, E. (2015). Uso y valoración de las TIC en el aprendizaje musical por los alumnos de los conservatorios de música profesional y superior de A Coruña: estudio de caso de uso de "IrealB" y "Band in a Box" en el aula de improvisación. (Tesis doctoral) Universidad Nacional de Educación a Distancia (UNED), España. Recuperado de: http://espacio.uned.es/fez/view/tesisuned:Educacion-Etimiraos 


\title{
INFORMACIÓN SOBRE LOS AUTORES
}

\section{Nuria Díez Latorre}

Universidad de Lleida

Titulada en Pedagogía del Lenguaje y la Educación Musical por el Conservatorio Superior de Música de Murcia. Diplomada en Magisterio Musical por la Universidad de Alicante. Máster en ELearning y Gestión del Conocimiento por la Universidad de Lérida. Músico profesional y docente en el ámbito de los conservatorios profesionales de música. Líneas de investigación: nuevas tecnologías en la educación musical, formación inicial docente en tecnologías.

\section{F. Xavier Carrera Farran} Universidad de Lleida

Doctor en Psicopedagogía. Premio extraordinario de Doctorado. Coordinador en la Universidad de Lleida del Programa de Doctorado Interuniversitario en Tecnología Educativa. Miembro del grupo de investigación COMPETECS (Competencias, Tecnología, Educación y Sociedad) de la Universidad de Lleida [2014 SGR 1611]. Sus líneas de investigación se centran en la Tecnología Educativa; la aplicación de metodologías activas en educación y en la formación basada en competencias.

http://webgrec.udl.es/webpages/personal/cat/000071 carrera.pip.udl.cat.html$$
\text { (c) (1) (9) }
$$

Los textos publicados en esta revista están sujetos a una licencia de Reconocimiento 4.0 España de Creative Commons. Puede copiarlos, distribuirlos, comunicarlos públicamente y hacer obras derivadas siempre que reconozca los créditos de las obras (autoría, nombre de la revista, institución editora) de la manera especificada por los autores o por la revista. La licencia completa se puede consultar en:Licencia Creative Commons Atribución-NoComercial-Compartir por igual 4.0 Internacional.
\end{abstract}

